\title{
Manipulative Reduction Splint External Fixation for the Treatment of Distal Radius Fractures
}

\author{
Yongming Tao \\ Lanzhou Orthopedics Hospital of Traditional Chinese Medicine; Lanzhou 730010, China.
}

Keywords: Manipulative reduction; External splint fixation; distal radius fracture; Clinical effect.

\begin{abstract}
Objective: To analyze the clinical effect of manual reduction splint external fixation in the treatment of distal radius fractures. Methods: From February 2014 to June 2017, 100 patients with distal radius fractures treated in our hospital were selected and divided into two groups according to the treatment method. Patients in the control group were treated by open reduction and internal fixation. The patients in the group were treated with manual reduction splint fixation and the clinical efficacy was compared between the two groups. Results: There were 35 patients, 11 patients, 3 patients, and 1 patient in the observation group with excellent, good, moderate, and poor respectively. The excellent and good rate was $92 \%$, which was higher than $70 \%$ of the control group. There was a statistically significant difference $(\mathrm{P}<0.05)$. The mean fracture healing time was shorter in the group than in the control group. The inclination of the palm and the ulnar deviation angle were smaller than those in the control group after treatment, and the shortening of the sacrum was greater than that in the control group. The difference was statistically significant $(\mathrm{P}<0.05)$. Conclusion: The clinical effect of manual reduction splint external fixation for distal radius fractures is significant and can be promoted.
\end{abstract}

\section{Introduction}

In recent years, the number of patients with distant radius fractures has increased significantly. This kind of fractures need to be treated with a reasonable way in time, because they usually caused by the energy trauma which may damage the smoothness of the articular surface and seriously affect the orthobiosis and work of the patients [1-2]. As a commonly used surgical method to treat this disease, although open reduction and internal fixation (hereinafter referred to as ORAIF) can obtain a certain curative effect, it may also lead to extensive tissue trauma, because the secondary surgery for removing the internal fixator will affect the prognostic stability of the fractured part to some extent [3-4]. In this context, the method manipulative reduction splint external fixation (hereinafter referred to as MRSEF) was adopted in this study, which not only obtained significant application effects, but also had many advantages such as simple operation, safety, etc. The relevant information is reported as follows.

\section{Data and Methods}

\subsection{Clinical Data.}

100 patients were selected from the patients with distal radius fractures treated in our hospital from February 2014 to June 2017, and all the selected patients signed informed consent voluntarily. They are divided into two groups according to the treatment method they accepted. The average age of patients in the observation group was $(59.56 \pm 3.69)$ years old, with a total of 50 cases: 39 of them are males and 11 females; the numbers of patients with fractures of type A, type $\mathrm{B}$ and type $\mathrm{C}$ were 22, 20 and 8 respectively; the fracture of 18 cases was caused by falling down, 12 by accidents, 13 by falling accidents and 7 by hitting from weights. The average age of patients in the control group was (58.99 \pm 4.29$)$ years old, with a total of 50 patients: 35 of them were males and 15 were females; the numbers of patients with fractures of type A, type $B$ and type $C$ were 21,19 and 10 respectively; the fracture of 19 cases was caused by falling down, 11 by accidents, 14 by falling accidents and 6 by hitting from weights. Comparing the two sets of basic data, the difference had the comparative value 
$(\mathrm{P}>0.05)$. The inclusion criteria: the fracture was diagnosed by $\mathrm{X}$-ray or $\mathrm{CT}$; there was obvious traumatism in the wrist joints, and the movements of wrist, forearm and finger were affected; there was a marked tenderness in the fractured end; the patient was in normal mental condition. Exclusion criteria: The patient was combined with other fractures that may affect the function of the affected limb; the one was combined with tendon, nerve and vascular injury in the affected limb; the one had a pathological fracture due to bone tumor, bone tuberculosis, etc.; the one had open or old fractures.

\subsection{Methods.}

ORAIF was applied in the control group. All patients were given brachial plexus block anesthesia and abducted the affected limbs, and what adopted was the approach of screwing in the distal radius palmaris. A longitudinal incision was made in the flexor carpi radialis, and the proximal end of the incision was extended by $7 \mathrm{~cm}$ to ensure that the distal end did not exceed the transverse stria of the wrist. The skin and subcutaneous tissue were incised in proper sequence and exposed to the deep layer between the radial artery and the radial flexor carpi, so as to fully expose the pronator. The pronator muscle was cut at the lateral edge of the humerus and pulled to the ulnar side to fully reveal the displaced bone and fractured end. The performer restored the broken end of the fracture under direct vision. Special attention was given to restore the inclination of the palm, ulnar deviation angle, the styloid height of the radius and length of the radius. Then the broken bone was fixed with T-shaped steel plate screws or LCP plate screws after being temporarily fixed with the Kirschner wire. These patients accepted treatments like pain relief, fluid rehydration and infection prevention according to their actual situation. Their X-ray films were reexamined on the second day after the operation. The patients were instructed to perform postoperative functional exercises after the fixation was confirmed.

MRSEF was applied in the observation group. Patients were instructed to accept surgical treatment at a sitting position. They were asked to abduce the shoulder joints to the maximum extent, the elbow remaining flexed at 90 degrees and the forearm at the neutral position. Firstly, the fractured limb was pulled and stretched for 5 minutes to correct the shortened deformity and promote the height recovery of the joint surface. Pressure was applied at the broken end of the fracture to correct the angulation displacement and the positive side. In the process of resetting, the moderate top-folding was implemented on the basis of the type of fracture and the angle of displacement. If the patient suffered a severely comminuted fracture, moderate distraction and extrusion were applied. The bone fricatives indicated that the fracture has been inserted and shifted. The performer could use the base part of both palms to evenly and softly tap and squeeze the fracture from the dorsal side of the palm, in order to squeeze the fractured fragments. In this process, special attention should be paid to giving even and gentle force. Then, under the condition of continuous traction, the wrist joints flexibly extended and cocked wrist to restore the flatness of the wrist joints. After obtaining a satisfactory reduction effect, the fixation was performed in the neutral position, palmar flexion position, dorsiflexion position and ulnar deviation position of the wrist respectively. The fractured limb was hung on the chest after being bandaged and fixed. X-ray examination was performed on the patients and the results of the review were used as a basis for reasonable adjustments.

\subsection{Observation Targets.}

The curative effect of the two groups of patients was evaluated in accordance with wrist function scoring system by Dienst [5-6]. The curative effect was rated as "poor" if the patient had following symptoms: the patient had continuous pain; the wrist flexion and extension loss exceeded 50 degrees; grip strength and joint functions significantly reduced and his/her daily life was obviously limited. The curative effect was rated as "moderate" if the patient had following symptoms: the patient suffered from frequent pain; the wrist flexion and extension loss was between 30 and 50 degrees; the grip strength and joint functions were slightly reduced and his/her work was slightly affected. The curative effect was assessed as "good" if the patient had following symptoms: the wrist flexion and extension loss was between 15 and 30 degrees; grip strength and joint functions were close to normal condition; pain appeared occasionally and only severe activities were limited. The curative effect was evaluated as "excellent" if the patient had following symptoms: the loss of wrist flexion and extension 
was less than 15 degrees; the grip strength and joint functions were completely normal condition; the joint activities were not limited and no pain appeared.

The two groups were compared in the mean fracture healing time, inclination of the palm, the ulnar deviation angle and shortening of the sacrum.

\subsection{Statistical Analysis.}

SPSS20.0 statistical software was used to analyze the data and enumeration data was tested by chi-square test, presented as ratio. Measurement data was analyzed by t-test and expressed as $(\bar{x} \pm \mathrm{s})$ and the difference was significant when $\mathrm{P}<0.05$.

\section{Results}

\subsection{The Clinical Efficacy Comparison of the Two Groups.}

For patients of the observation group after treatment, 35 enjoyed excellent curative effects, 11 enjoyed good effects, 3 enjoyed moderate effects and curative effects of 1 patient was poor. Excellent and good rate was $92 \%$, higher than $70 \%$ of the control group; the difference was statistically significant $(\mathrm{P}<0.05)$, as shown in Table 1:

Table 1. The clinical efficacy comparison of the two groups [n (\%)]

\begin{tabular}{ccccccc}
\hline Group & $\begin{array}{c}\text { cases } \\
(\mathrm{n})\end{array}$ & Excellent & Good & Moderate & Poor & $\begin{array}{c}\text { Excellent and good } \\
\text { rate }\end{array}$ \\
\hline Control group & 50 & $20(40.00)$ & $\begin{array}{c}15 \\
(30.00)\end{array}$ & $10(20.00)$ & $\begin{array}{c}5 \\
(10.00)\end{array}$ & $35(70.00)$ \\
$\begin{array}{c}\text { Observation } \\
\text { group }\end{array}$ & 50 & $35(70.00)$ & $\begin{array}{c}11 \\
(22.00)\end{array}$ & $3(6.00)$ & $1(2.00)$ & $46(92.00)$ \\
X2 & & & & & 7.8622 \\
P & & & & & & 0.0050 \\
\hline
\end{tabular}

\subsection{The Clinical Index Comparison of the Two Groups.}

The mean fracture healing time was shorter in the group than in the control group. The inclination of the palm and the ulnar deviation angle were smaller than that in the control group after treatment, and the shortening of the sacrum was greater than that in the control group. The difference was statistically significant $(\mathrm{P}<0.05)$, as shown in Table 2 .

Table 2. The clinical index comparison of the two groups $\left(\bar{x}_{ \pm \mathrm{s}}\right)$

\begin{tabular}{ccccc}
\hline Group & $\begin{array}{c}\text { Fracture healing } \\
\text { time (weeks) }\end{array}$ & $\begin{array}{c}\text { Inclination of the } \\
\text { palm (degrees) }\end{array}$ & $\begin{array}{c}\text { Ulnar deviation } \\
\text { angle (degrees) }\end{array}$ & $\begin{array}{c}\text { Shortening of the } \\
\text { sacrum (mm) }\end{array}$ \\
\hline $\begin{array}{c}\text { Control group } \\
(\mathrm{n}=50)\end{array}$ & $7.89 \pm 1.59$ & $6.59 \pm 4.45$ & $13.65 \pm 4.98$ & $1.45 \pm 1.02$ \\
Observation & $6.02 \pm 1.22$ & $4.10 \pm 3.95$ & $12.02 \pm 1.26$ & $1.99 \pm 2.99$ \\
group $(\mathrm{n}=50)$ & 6.5979 & 2.8877 & 2.2437 & 3.9562 \\
$\mathrm{t}$ & 0.0000 & 0.0048 & 0.0271 & 0.0000 \\
$\mathrm{P}$ &
\end{tabular}

\section{Discussion}

The distal radius fracture is a common type of fractures occurring within $3 \mathrm{~cm}$ of the articular surface of the distal radius; the elderly and adolescents with osteoporosis are the prominent population stricken by this disease [7-8]. The disease can have a direct effect on the wrist functions of the patient. If it is not treated with a correct and reasonable method in time, the wrist joint may suffer from chronic pain, weakened grip and stiffness. The key to the treatment of this disease lies in restoring the anatomy of the wrist to restore the function of the wrist [9-10]. In this study, two groups of patients with distal radius fractures were treated with ORAIF and MRSEF respectively. After 
treatment, the excellent and good rate in the observation group was $92 \%$ which was higher than $70 \%$ in the control group $(\mathrm{P}<0.05)$. The mean fracture healing time was shorter in the group than in the control group; the inclination of the palm and the ulnar deviation angle were smaller than those in the control group after treatment, and the shortening of the sacrum was greater than the control group, $\mathrm{P}<0.05$. It can be seen that, compared with the method ORAIF, MRSEF is more effective in the treatment of tibial fractures, because it is able to effectively improve the wrist function of patients and promote fracture healing. First of all, this may be because MRSEF does not have a significant effect on the patient's functional limb exercise, so he/she can perform functional exercise during fracture healing. Secondly, compared with ORAIF, the fixation method is more convenient and does not require secondary surgery to remove the fixture, which is beneficial for reducing the patients' pain. Although the application of MRSEF is effective, appropriate splints should be selected and placed strictly in the order of "front-back-inside-outside" when treating patients with this method. If the patient suffers a Colles fracture, it is necessary to ensure that the dorsal and radialis splints exceed the wrist joint and the wrist joint is fixed in the ulnar deviation position. If the patient suffers a Chance fracture, it is necessary to ensure that the splint on the dpalmaris exceeds the wrist joint and the wrist joint is fixed in the dorsiflexed position. In addition, it is necessary to guide the patient to carry out rehabilitation training according to his/her actual situation of the patient, so as to effectively promote fracture healing.

In summary, the application of MRSEF on distal radical fractures has obvious clinical effect and has significant promotion value.

\section{References}

[1]. Mo Bingeing, Yin Dong, Huang Yu, et al. Efficacy Contrast of Manual Splint Fixation and External Fixation for the Treatment of Distal Radius Fractures of the Elderly [J]. Shandong Medical Journal, 2016, 56(44): 89-91.

[2]. DU Liang, Chen Key, Hu Wuxing, et al. Meta-Analysis on Treatment of Distal Radius Fractures with Closed Reduction and External Fixation or Open Reduction and Internal Fixation [J]. Heilongjiang Medical Journal, 2015, 39(11):1213-1216.

[3]. Fu Lice, Yang Ruin, Xu Weidong. Influence of external fixator combined with Kirschner wires internal fixation on the recovery of wrist function in distal radius fractures [J]. Hainan Medical Journal, 2017, 28(23):3843-3846.

[4]. Guy Kaesong, Zhang Hainan, Huang Lin, et al. Analysis of Anatomic Parameters of Articulatio Radiocarpea and Wrist Joint Function after Three Types of External Fixation for Unstable Distal Radius Fractures in the Elderly [J]. Chinese Journal of Bone and Joint Injury, 2017, 32(5): 541-543.

[5]. Liu Wei, Zhu Men, Tang Wanwan, et al. Effect and Safety of Two Kinds of Different Reduction Fixation Methods in Treatment of Unstable Distal Radius Fractures [J]. Medical \& Pharmaceutical Journal of Chinese People's Liberation Army, 2017, 29(10): 42 -45.

[6]. Chen Min, Li Tanguy, Huang Guangping, et al. Comparison of the curative effect between over-elbow splint and traditional small splint fixation in the treatment of comminuted Colles fracture [J]. China Journal of Orthopaedics and Traumatology, 2017, 30(3):222-226.

[7]. Chen Jialing, Zhang Long Jun, $\mathrm{Cu}$ Yong, et al. Clinical research of percutaneous K wires fixation after manipulative reduction combining with gypsum or splint fix-ation for treatment of the Barton fractures of aged people [J]. China Journal of Orthopaedics and Traumatology, 2016, 29(1): 8-12. 
[8]. Wei Layout, Zhang Hogweed, Zhao Gang. Sodium aescinate gel alleviates hand swelling in elderly patients with distal radius fracture [J]. Chinese Journal of General Practitioners, 2016, 15(10):789-791.

[9]. Xian Zhengzhou, Dong Xia, Yuan Rongxia, et al. Comparative Study of Manipulative Reduction Splint External Fixation and Open Reduction and Kirschner Wire Fixation for Treatment of Distal Radius Fractures in Children [J]. Journal of Chengdu University of Traditional Chinese Medicine, 2017, 40 (3): 72-75.

[10]. Wang Jain, Wang Gang, Gejilertu, et al. Comparison of Efficacy of External Fixation with Limited Internal Fixation and Open Reduction Internal Fixation for the Treatment of Elderly Distal Radius Fractures [J]. Chinese Journal of Orthopaedic Trauma, 2014, 16 (10): 912-914. 